\title{
EROSÃO EM SULCOS EM DIFERENTES PREPAROS E ESTADOS DE CONSOLIDAÇÃO DO SOLO ${ }^{(1)}$
}

\author{
M. J . SCHÄFER ${ }^{(2)}$, J . M. REICHERT (3), E. A. CASSOL ${ }^{(4)}$, \\ F.L.F.ELTZ(3) \& D.J . REINERT(3)
}

\begin{abstract}
RESUMO
O preparo e a consolidação do solo alteram a sua capacidade em resistir à erosão em sulcos. Com o objetivo de estudar a erosão em sulcos em diferentes preparos e consolidação do solo, conhecer o diâmetro mediano dos sedimentos transportados e determi nar a erodi bilidade em sulcos $(\mathrm{Kr})$ e a tensão crítica de cisalhamento $(\tau c)$ do solo, foi realizado um experimento no campo, em 1997/98, em um Argissolo Vermelho-Amarelo distrófico arênico. 0 delineamento experimental foi inteiramente casualizado com seis repetições. Os tratamentos constaram de: preparo convencional recente (CR), preparo convencional consolidado (consolidação de dois meses) (CC), plantio direto sem palha (PDS) e plantio direto com palha (PDC, 94\% de cobertura). Usou-se chuva simulada de intensidade constante $\left(65 \mathrm{~mm} \mathrm{~h}^{-1}\right)$ até escoamento aproximadamente constante de água no solo. E m seguida, na extremidade superior do sulco, foram adicionadas descargas líquidas $(\mathrm{Q})$ crescentes de $0,0002 \mathrm{~m}^{3} \mathrm{~s}^{-1}$ até $0,0010 \mathrm{~m}^{3} \mathrm{~s}^{-1}$, para os tratamentos CR e CC, e de $0,0004 \mathrm{~m}^{3} \mathrm{~s}^{-1}$ até $0,0020 \mathrm{~m}^{3} \mathrm{~s}^{-1}$, para os tratamentos PDS e PDC, sendo as amostras coletadas na parte inferior de cada sulco. As parcelas foram del imitadas por chapas metálicas cravadas no solo no sentido do declive (0,20 m de largura por 6,00 m de comprimento). 0 valor de $\mathrm{Kr}$ determinado foi de $0,012 \mathrm{~kg} \mathrm{~N}^{-1} \mathrm{~s}^{-1}$ e o $\tau \mathrm{c}$ foi de 2,61 $\mathrm{N} \mathrm{m}^{-2}$. A desagregação, as perdas de solo e o diâmetro mediano dos sedimentos apresentaram a seguinte seqüência em magnitude: CR, CC, PDS e PDC, particularmente nas maiores $Q$. 0 regime de escoamento foi turbulento supercrítico, com exceção da primeira $Q$ aplicada, onde o regime foi laminar subcrítico, para o PDC, graças à presença de resíduos culturais, e lami nar supercrítico, para os demais tratamentos. A consolidação e a cobertura do solo alteram o regime do escoamento e reduzem a erosão em sulcos e seus efeitos são complementares.
\end{abstract}

Termos de indexação: erodibilidade em sulcos, plantio direto, tamanho de sedi mento, perda de solo e água, hidrologia do solo.

\footnotetext{
(1) Parte da Tese de Mestrado do primeiro autor, apresentada ao Programa de Pós Graduação em Agronomia, U niversidade F ederal de Santa Maria - UFSM. Pesquisa parcial mente financiada com recursos da FAPE RGS e do Pronex-CNPq/FI NEP. Recebido para publicação em novembro de 1999 e aprovado em janeiro de 2001.

(2) Engenheiro-Agrônomo, MSc. Rua 5 de Março, 83. CEP 97110-000 Santa Maria (RS).

(3) Professor do Departamento de Solos, Universidade Federal de Santa Maria - UFSM. CEP 97105-900 Santa Maria (RS). Pesquisador do CNPq. E-mail: reichert@ccr.ufsm.br

(4) Professor do Departamento de Solos, Universidade Federal do Rio Grande do Sul - UFRGS. CEP $90001-970$ Porto Alegre (RS). Pesquisador do CNPq.
} 


\title{
SUMMARY: RILL EROSION UNDER DIFFERENT SOIL TILLAGE METHODS AND SOIL CONSOLIDATION
}

\begin{abstract}
Soil tillageand consolidation modify soil resistanceto rill erosion. Thus, an experiment was carried out on a Hapludulf, under field conditions in 1997/ 98, to study rill erosion under different tillage methods and soil consolidation, to eval uatethe sediment size and to determine rill erodibility $(\mathrm{Kr})$ and critical shear stress $(\tau c)$. The experiment was completely randomized with six replications, using thefoll owing treatments: recent conventional tillage (RCT), two-month consolidated conventional tillage (CCT), no-tillage with mulch (NTM) with $94 \%$ surface coveragewith crop residues, and no-tillagewith a baresoil surface(NTB). A constant simulated rainfall of $65 \mathrm{~mm} \mathrm{~h}^{-1}$ was applied until a steady-state runoff rate was reached. Afterwards, extra inflows were applied at rates ranging from $0.0002 \mathrm{~m}^{3} \mathrm{~s}^{-1}$ to $0.0010 \mathrm{~m}^{3} \mathrm{~s}^{-1}$, for RCT and CCT, and from $0.0004 \mathrm{~m}^{3} \mathrm{~s}^{-1}$ to $0.0020 \mathrm{~m}^{3} \mathrm{~s}^{-1}$. Theplots $(0.2 \mathrm{~m}$ wide by $6.0 \mathrm{mlong}$ ) were confined by metal borders along theslope. The Kr was $0.012 \mathrm{~kg} \mathrm{~N}^{-1} \mathrm{~s}^{-1}$ and $\tau \mathrm{c}$ was $2.61 \mathrm{~N} \mathrm{~m}^{-2}$. Soil detachment, soil loss and sediment size had the following order RCT , CCT, NTM and NTB, particularly for thehigher flows. Flow regimewas turbulent and supercritical, except for the lowest inflow, where the flow was laminar and subcritical for NTM, due to the mulch, and supercritical laminar for the remaining treatments. Soil consol idation and surface coveragemodify fl ow regimeand reduceerosi on, and their effects arecomplementary.
\end{abstract}

Index terms: rill erodi bility, no-tillage, sediment size, soil and water losses, soil hydrology.

\section{INTRODUÇÃO}

A erosão hídrica do solo é causada por agentes erosivos, como a precipitação pluvial e o escoamento superficial, e consiste do destacamento, transporte e deposição de partículas do solo. No processo de erosão em sulcos, o agente responsável pela desagregação etransporte dos sedimentos em sulcos é o escoamento concentrado (Meyer et al., 1975; Foster, 1982). A desagregação ocorre por ação das forças de cisal hamento atuantes no contorno sólido (interface água-superfície do solo), sendo o transporte dos sedimentos influenciado pela descarga líquida e pela velocidade do escoamento (Foster, 1982).

A erosão em sulcos ocorre somente quando as forças de cisal hamento do escoamento superam as forças coesivas do solo, momento em que ocorre a tensão crítica de cisalhamento (F oster, 1982). Portanto, a erosão em sulcos é uma resultante da interação da capaci dade do escoamento em erodir e a capacidade do solo em resistir às forças de desagregação e transporte pela água. N earing et al . (1991) observaram que a taxa de desagregação dos solos foi adequadamente descrita pela tensão cisalhante $(\tau)$ ou pela potência $(\Omega)$ do escoamento. Entretanto, em algumas situações, $\Omega$ pode ser um melhor estimador da desagregação em sulcos que $\tau$ (Elliot \& Laflen, 1993). A potência do escoamento é a quantidade de energia do escoamento por unidade de tempo e de área (Bagnold, 1977).
A erodibilidade em sulcos $(K r)$ de um solo é representada pela susceptibilidade à desagregação pel o escoamento superficial concentrado. O sol o pode ser mais ou menos erodível, conforme suas características físicas, químicas e mineralógicas, e a erosão muito afetada pelo preparo do solo (F oster, 1982).

O revolvimento aumenta a susceptibilidade à erosão e reduz a tensão crítica de cisalhamento do solo, enquanto a consolidação do solo produz um efeito inverso (F oster, 1982). No contexto agrícola, a consolidação é definida como um aumento na estabilidade estrutural do solo, proveniente da tensão efetiva (compressão) induzi da pel o potencial matricial durante a redistribuição da água no solo após a chuva (Nearing et al., 1988b). Essa consol idação al tera as propriedades do sol o durante o ano, tais como: densidade e porosidade do solo, retenção e infiltração de água, assim como a susceptibilidade à erosão e tensão crítica de cisal hamento do solo (N earing et al., 1988a).

Vários pesquisadores (Cogo et al., 1984; Brown $\&$ Norton, 1990; West et al., 1991; N orton \& Brown, 1992) documentaram os benefícios da cobertura vegetal na redução da desagregação do solo em métodos conservacionistas. Manter os resíduos na superfície do sol o por vários anos em sistema plantio direto apresenta grandes vantagens, como a redução do impacto da gota de chuva e selamento/ encrostramento superficial (Alves \& Cabeda, 1999; Barcel os et al., 1999), e diminuição da desagregação 
do sol o etransporte dos sedimentos (Cogo et al., 1984; Alves et al., 1995; Bertol et al., 1997).

Os resíduos na superfície do solo reduzem a tensão cisalhante do escoamento que atua sobre o solo, pois essa tensão passa a ser dividida entre os resíduos presentes e o próprio solo (F oster, 1982). Entretanto, os resíduos vegetais soltos na superfície do solo são eficientes na erosão em sulcos somente até o momento em que o escoamento formado conseguir transportá-los, havendo fal ha dos resíduos decorrentes de grandes declives ou grandes escoamentos de água ou da formação de sul co sob os resíduos (Bertol et al., 1997). Em um experimento de campo, estudando os efeitos da erosão em sulcos sobre a consol idação do sol o e resíduos incorporados, Brown et al. (1990) observaram uma desagregação duas vezes mai or notratamento com sol o preparado recentemente em relação ao consol idado durante um ano.

Valores para a erodi bilidade em sul cos $(\mathrm{Kr})$ e para a tensão crítica de cisalhamento $(\tau c)$ para trinta e seis solos foram determinados experimentalmente por Elliot et al. (1989) nos Estados Unidos. O parâmetro mais importante na regressão para predizer o $\mathrm{Kr}$ foi a matéria orgânica seguida pelo parâmetro textural M. No Brasil, poucos estudos experimentais no campo têm adotado o modelo WEPP (Giasson \& Cassol, 1996; Braida \& Cassol, 1996), sendo insuficientes para estabel ecer relações entre $\mathrm{Kr}$ e $\tau \mathrm{c}$ com propriedades dos solos. Outrossim, estudos sobre processos e mecânica da erosão do solo usando princípios e model os fundamentais de erosão são também pou cos. A contribuição da consolidação do solo, isolada ou conjuntamente com a cobertura morta, na redução da erosão do solo não está total mente estabel ecida.

Assim, os objetivos deste experimento foram: (a) quantificar a erodibilidade em sulcos $(\mathrm{Kr})$ ea tensão crítica de cisal hamento do solo $(\tau c)$, (b) determinar parâmetros da erosão e do escoamento em sulcos em diferentes sistemas de manejo e consol idação do solo, e (c) avaliar o efeito de diferentes sistemas de manejo e consol idação do sol o no diâmetro mediano dos sedimentos transportados em sulcos.

\section{MATERIAL E MÉTODOS}

\section{Tratamentos e aplicação das chuvas e descargas líquidas}

O experimento foi realizado na área experimental do Departamento de Sol os da Universidade F ederal deSanta Maria (UFSM), Santa Maria (RS), noverão de 1997/98. O solo estudado foi um Argissolo Vermelho-Amarelo distrófico arênico (EMBRAPA, 1999) da unidade de mapeamento São Pedro.

O delineamento experimental foi o inteiramente casual izado, com quatro tratamentos eseis repetições.
Os tratamentos foram: preparo convencional recente (CR), preparo convencional consolidado (CC, consolidação por dois meses), plantio direto sem presença de pal ha (PDS, palha removida) e plantio direto com presença de palha (PDC, com 94\% de cobertura do solo). Algumas características do solo, detal hes do histórico da área e estabel ecimento dos tratamentos encontram-se em Schäfer et al. (2001).

As parcelas de erosão foram delimitadas por chapas metálicas galvanizadas cravadas no sol o, que continham, na parteinferior, uma cal ha coletora para a amostragem do escoamento. Com a delimitação dos canais com as chapas metálicas (King, 1992), conseguiu-se escoamento concentrado sem necessidade de pré-formação de sul cos (F oster et al., 1984; Giasson \& Cassol, 1996). As dimensões das parcelas de erosão foram de $0,20 \mathrm{~m}$ de largura e 6,00 m de comprimento, ficando a maior dimensão no sentido do maior declive.

As chuvas foram aplicadas com um simulador estacionário de bicos múltiplos e oscilantes (N orton \& Brown, 1992), instalado a uma altura de $2,45 \mathrm{~m}$ da superfície do sol o e com pressão de saída da água nos bicos mantida em 41,4 kPa. A chuva de $65 \mathrm{~mm} \mathrm{~h}^{-1}$ foi aplicada durante o tempo necessário para levar o solo a escoamento aproximadamente constante. Esse tempo variou entre 30 e $50 \mathrm{~min}$, dependendo da umidade inicial do solo. A intensidade de chuva efetiva foi monitorada com o auxílio de pluviômetros instalados ao lado da parcela de erosão.

Imediatamente após a chuva, para simular diferentes taxas de escoamento ou descargas líqui das (Q), foram adicionadas vazões extras de 0,0002; 0,$0004 ; 0,0006 ; 0,0008$ e $0,0010 \mathrm{~m}^{3} \mathrm{~s}^{-1}$, para os tratamentos CR e CC, e de 0,0004; 0,0008; 0,0012; 0,0016 e $0,0020 \mathrm{~m}^{3} \mathrm{~s}^{-1}$, para os tratamentos PDS e PDC. As referidas $Q$ foram medidas com um rotâmetro. As coletas de escoamento foram realizadas com o auxílio de uma calha colocada na porção inferior de cada sulco.

Durante a chuva, foram col etadas amostras de escoamento de $10 \mathrm{em} 10$ min ecada pote permaneceu o tempo suficiente para encher. Ao final de cada chuva, foi coletada uma amostra em balde de três litros para determinação do tamanho de sedimentos erodidos e medida a temperatura da água para cálculos hidráulicos. Os recipientes utilizados na captação foram potes plásticos com capacidade de um litro.

Por ocasião da aplicação da descarga líquida extra, foram col etadas três amostras do escoamento com potes de um litro por descarga, para determinar as perdas de água, solo, infiltração e concentração de sedimento, e uma ao final de cada descarga, em balde detrêslitros, para determinar otamanhodesedimento erodido. Cada descarga permaneceu no sulco o tempo estritamente necessário (aproximadamente $3 \mathrm{~min}$ ) para estabilizar o escoamento em cada descarga e coletar as amostras, medir a profundidade do 
escoamento e determinar a velocidade do escoamento. I mediatamente após, adicionou-seoutra descarga no mesmo sulco. O tempo de col eta de cada amostra foi cronometrado.

\section{Determinações e cálculos de parâmetros de erosão}

As amostras de escoamento col etadas no campo foram levadas ao laboratório e pesadas para determinar o teor de solo e água. As amostras coletadas em baldes no final de cada chuva foram peneiradas em água para medir o tamanho de sedimentos erodidos pelos parâmetros diâmetro médio geométrico (DMG) e diâmetro mediano $\left(D_{50}\right)$, conforme procedimentos descritos em Schäfer et al . (2001).

A taxa de desagregação ( $\mathrm{Dr}, \mathrm{kg} \mathrm{m}^{-2} \mathrm{~s}^{-1}$ ) foi calculada por: $\mathrm{Dr}=$ descarga sól ida $\left(\mathrm{kg} \mathrm{s}^{-1}\right) /$ área total $\left(\mathrm{m}^{2}\right)$ do sulco. A área do sulco (A) foi obtida multiplicando-se a largura do escoamento, medida no campo, pelo comprimento do canal, que foi de 5,90 m. A descarga sólida (qs) foi encontrada pela fórmula: qs $=Q \mathrm{C}$, sendo: $\mathrm{Q}=$ descarga líquida $\left(\mathrm{m}^{3} \mathrm{~s}^{-1}\right)$ eC = concentração de sedimento $\left(\mathrm{kg} \mathrm{m}^{-3}\right): \mathrm{Q}=$ massa deágua ( $\mathrm{kg}) /$ tempo (s) eC =massa desolo $(\mathrm{kg}) /$ vol ume de água $\left(\mathrm{m}^{-3}\right)$.

A área da seção transversal do escoamento $\left(A, m^{2}\right)$ foi calculada por: $\mathrm{A}=\mathrm{Q} N_{\mathrm{m}}\left(\mathrm{Q}=\right.$ descarga líquida $\left(\mathrm{m}^{3} \mathrm{~s}^{-1}\right)$ e $V_{m}=$ velocidade média do escoamento $\left(\mathrm{m} \mathrm{s}^{-1}\right)$. A vel ocidade média do escoamento $\left(V_{m}, \mathrm{~m} \mathrm{~s}^{-1}\right)$ foi obtida por: $\mathrm{V}_{\mathrm{m}}=$ velocidade na superfície do escoamento multiplicado por 0,74 (King, 1992), que é o fator de correção atribuído à diminuição da vel ocidade com a profundidade do escoamento decorrente do atrito com o solo. A velocidade na superfície do escoamento foi determinada com o uso de uma solução traçadora de azul de metileno.

A tensão de cisalhamento do escoamento $\left(\tau, \mathrm{N} \mathrm{m}^{-2}\right)$ foi obtida pela equação: $\tau=\operatorname{Rh} S \gamma$, enquanto a potência do escoamento $\left(\Omega, \mathrm{N} \mathrm{m}^{-1} \mathrm{~s}^{-1}\right)$ foi calculada pela fórmula: $\Omega=\gamma \mathrm{Q}$ S, em que: $\mathrm{Rh}=$ raio hidráulico (m), $\mathrm{S}=$ declividade do sulco $\left(\mathrm{m} \mathrm{m}^{-1}\right), \gamma=$ peso específico da água $\left(\mathrm{N} \mathrm{m}^{-3}\right)$ e $\mathrm{Q}=$ descarga líquida $\left(\mathrm{m}^{3} \mathrm{~s}^{-1}\right)$. O raio hidráulico $(\mathrm{Rh})$ foi calculado pela fórmula: $\mathrm{Rh}=\mathrm{A}$ /perímetro mol hado. A largura e a profundidade do escoamento foram determinadas com o auxílio de uma régua. A altura foi medida entre um ponto fixo acima do escoamento e a superfície do escoamento de água.

A tensão cisalhante do escoamento no sulco, quando existe presença de resíduos culturais, não é exercida toda diretamente sobre o solo, pois parte dela é dissipada sobre o resíduo. Calculando a diferença entrea perda de carga no PDC ea doPDS, que apresentava as mesmas condições de solo mas sem a presença dos resíduos culturais na superfície, obteve-se a tensão exercida sobre a palha. Para o cálculo da tensão cisalhante exercida somente no solo, usou-se a expressão $\tau_{\text {solo }}=\tau_{\text {total }}\left(f_{\text {no sold }} d f_{\text {total }}\right)$ (Foster, 1982; King, 1992), em que: $\tau_{\text {solo }}=$ tensão cisalhante do escoamento exclusivamente sobre o solo sem a pal ha no PDC; $f_{\text {no solo }}=$ perda de carga no PDS; $f_{\text {total }}=$ perda de carga total noPDC na presença da palha e $\tau_{\text {total }}=$ tensão cisalhante sobre o solo e palha no PDC.

O fator perda de carga de Darcy-Weisbach $(f)$, o número de Reynolds (Re) e o número de $F$ roude $(F)$ foram calculados conforme Chow (1959). O $f$ é um fator que descreve a resistência ao escoamento, o Re éumíndice deturbulência doescoamentoeo $\mathrm{F}$ indica se há formação de ondas gravitacionais (Chow, 1959) e são todos adimensionais.

A erodibilidade em sulcos $(\mathrm{Kr})$ e a tensão crítica de cisal hamento (c) for am calculadas pela equação: $\mathrm{Dr}=\mathrm{Kr}(\tau-\tau \mathrm{c})$, sendo: $\mathrm{Dr}=$ taxa de desagregação do sol o em sulcos $\left(\mathrm{kg} \mathrm{m}^{-2} \mathrm{~s}^{-1}\right), \mathrm{Kr}=$ erodi bilidade do solo em sulcos $\left(\mathrm{kg} \mathrm{N}^{-1} \mathrm{~s}^{-1}\right), \tau=$ tensão cisalhante do escoamento $\left(\mathrm{N} \mathrm{m}^{-2}\right)$ e $\tau \mathrm{c}=$ tensão crítica de cisal hamento ( $\mathrm{N} \mathrm{m}^{-2}$ ) (F oster, 1982). Para que essa equação possa ser usada na determinação da Dr, a relação Dr/Dc deve tender à unidade (Elliot, 1988; King, 1992). Isso ocorre quando a carga de sedimento (G) no escoamento é muito pequena se comparada com a capacidade de transporte (Tc) do escoamento, podendo-se assumir que o sedimento em transporte não reduz a desagregação (Elliot, 1988).

Alternativamente, expressou-se a equação anterior como $\mathrm{Dr}=\mathrm{Kr}_{\Omega}(\Omega-\Omega \mathrm{C})$, em que: $\Omega=$ potência do escoamento $\left(\mathrm{N} \mathrm{m}^{-1} \mathrm{~s}^{-1}\right), \Omega \mathrm{C}=$ potência crítica do escoamento $\left(\mathrm{N} \mathrm{m}^{-1} \mathrm{~s}^{-1}\right)$ e $\mathrm{Kr}_{\Omega}=$ parâmetro de erodibilidade em sulcos $\left(\mathrm{kg} \mathrm{N}^{-1} \mathrm{~m}^{-1}\right)$, calculado com base na desagregação do solo e da potência do escoamento(Elliot, 1988; King, 1992; Elliot \& Laflen, 1993).

Para cal cular a carga de sedimento $\left(\mathrm{G}, \mathrm{kg} \mathrm{m}^{-1} \mathrm{~s}^{-1}\right)$, dividiu-se a descarga sólida $\left(\mathrm{kg} \mathrm{s}^{-1}\right)$ pela largura do escoamento $(m)$ em cada descarga. A capacidade de transporte ( $\mathrm{Tc}, \mathrm{kg} \mathrm{m}^{-1} \mathrm{~s}^{-1}$ ) do escoamento foi estimada pela equação de Yalin (J ulien, 1988), conforme procedimentos relatados por Elliot (1988).

Para analisar os dados qualitativos, usaram-sea análise de variância e o teste de médias (Duncan $5 \%$ ) e, para os dados quantitativos, realizou-se a análise deregressão para ajuste de equações lineares enão-lineares, usando o pacote estatísticoSAS (SAS Institute, 1988).

\section{RESULTADOS E DISCUSSÃO}

\section{Chuva simulada}

A menor descarga líquida constante $\left(Q_{c}\right)$ (Quadro 1) durante a chuva ocorreu no solo preparado e com dois meses de consolidação (CC), 
Quadro 1. Descarga líquida constante $\left(Q_{c}\right)$, descarga sólida constante $\left(q s_{c}\right)$ e concentração constante de sedimento $\left(C_{c}\right)$, considerando os di fer entes preparos do solo e consolidação durante a chuva si mulada de $65 \mathrm{~mm} \mathrm{~h}^{-1}$

\begin{tabular}{lccc}
\hline \multicolumn{1}{c}{ Tratamento } & Qc & $\mathbf{q s}$ & $\mathbf{C}_{\mathbf{c}}$ \\
\hline & $\mathrm{m}^{3} \mathrm{~s}^{-1}$ & $\mathrm{~kg} \mathrm{~s}^{-1}$ & $\mathrm{~kg} \mathrm{~m}^{-3}$ \\
Convencional recente (CR) & $0,000015 \mathrm{a}$ & $0,000218 \mathrm{a}$ & $14,78 \mathrm{a}$ \\
Convencional consolidado (CC) & $0,000011 \mathrm{~b}$ & $0,000153 \mathrm{ab}$ & $12,96 \mathrm{a}$ \\
Plantio direto sem palha (PDS) & $0,000015 \mathrm{a}$ & $0,000104 \mathrm{~b}$ & $7,23 \mathrm{~b}$ \\
Plantio direto com palha (PDC) & $0,000015 \mathrm{a}$ & $0,000010 \mathrm{c}$ & $0,64 \mathrm{c}$ \\
\hline
\end{tabular}

Médias seguidas pela mesma letra, na coluna, não diferem pelo teste de Duncan a 5\%.

não havendo redução na $Q_{c}$ com o sistema plantio direto com (PDC) ou sem (PDS) cobertura em relação ao preparo convencional recente(CR). Em contraste, Cassol \& Lima (1998) verificaram uma redução de $53 \%$ na taxa de descarga líquida do plantio direto, indicando que a hidrologia do sol o sob plantio direto depende da qualidade do espaço poroso do solo. A menor $Q_{c}$ em sulcos no tratamento CC está em consonância com a baixa taxa constante de escoamento de água em entressulcos, obtida para esse tratamento (Schafer et al., 2001).

A descarga sólida (qs) e a concentração de sedimentos (C) constante foram as menores no tratamento com cobertura vegetal (PDC), graças à interceptação das gotas da chuva e dissi pação da sua energia cinética, e maiores no CR e CC, graças ao impacto das gotas de chuva na superfície sem cobertura erecentemente revolvida, sem expressarse o efeito da consol idação do solo.

A concentração constante de sedimento $\left(C_{c}\right)$ no escoamento (Quadro 1) reflete as quanti dades de sol o transportadas quando o escoamento se estabilizou. As maiores $C_{c}$ ocorreram no $C R$ e no $C C$, enquanto a menor $\mathrm{C}_{\mathrm{c}}$ ocorreu no PDC considerando as baixas taxas de desagregação do solo e do transporte de sedimento, resultantes da proteção da cobertura pelos resíduos vegetais e associadas à resistência conferida pelos processos de consolidação e agregação do solo.

O menor diâmetro médio geométrico (DMG) e mediano $\left(D_{50}\right)$ dos sedimentos erodidos pelo escoamento decorrente da chuva (Quadro 2) ocorreu no PDC, indicando serem os solos com adequada agregação e cobertura vegetal mais resistentes à erosão e serem os sedimentos de menor tamanho transportados. Comparando somente os solos preparados convencional mente, $0 \mathrm{DMG}$ e o $\mathrm{D}_{50}$ foram menores no CR que no CC, no qual a consolidação aumentou a estabilidade de agregados do solo (Schäfer et al., 2001), aumentando a sua resistência ao impacto das gotas da chuva.
Quadro 2. Diâmetro médio geométrico (DMG) e mediano $\left(D_{50}\right)$ dos sedimentos no escoamento coletado durante o período da chuva nos sulcos, considerando os diferentes sistemas de preparo do solo e consolidação

\begin{tabular}{|c|c|c|}
\hline Tratamento & DMG & $\mathbf{D}_{50}$ \\
\hline & \multicolumn{2}{|c|}{$\mathrm{mm}$} \\
\hline Convencional recente $(\mathrm{CR})$ & $0,12 \mathrm{~b}$ & $0,07 \mathrm{~b}$ \\
\hline Convencional consolidado (CC) & $0,21 a$ & 0,11 a \\
\hline Plantio direto sem palha (PDS) & $0,10 \mathrm{~b}$ & 0,11 a \\
\hline Plantio direto com palha (PDC) & $0,05 c$ & $0,06 \mathrm{~b}$ \\
\hline
\end{tabular}

Médias seguidas pela mesma letra, na coluna, não diferem pelo teste de Duncan a 5\%.

\section{Descargas líquidas extras aplicadas no sulco}

Com a descarga líquida (vazão) extra aplicada no sulco, no tratamento CR, as descargas sól idas (qs) e as taxas de desagregação (Dr) foram crescentes até à descarga mais alta, com el evada concentração de sedimentos no escoamento (Figura 1 ). No CC e PDS, as curvas para taxa de desagregação e concentração de sedimento tiveram um ponto de máxima e um decréscimo posterior.

Esse fato parece estar relacionado com a existência de uma camada superficial mais erodível (correspondenteà profundidade de preparo do solo), a qual é removida por escoamentos mais intensos, e de uma camada subjacente mais compactada (F oster, 1982), decorrente do não-revolvimento com pé-dearado, que resiste ao destacamento de partículas de solo. No PDC, a taxa de desagregação (Dr) com a descarga líquida (Q) foi significativa, porém com pequeno incremento da $\mathrm{Dr}$ com a Q, considerando a cobertura do solo proporcionada pelos resíduos vegetais, a consolidação ea agregação que reduziram a desagregação e as perdas de solo. 
Em estudos similares, Norton \& Brown (1992) encontraram que a consolidação reduziu em $72 \%$ a erosão em sulcos em dois solos (franco-siltoso e argilo-siltoso) consolidados por até oito anos, comparados aos recém-preparados, mas não houve diferença entre os solos consolidados por diferentes durações. Brown \& Norton (1994) encontraram reduções de $88 \%$ na perda de solo, devidas à consolidação de oito anos, e mais $4 \%$, à cobertura (45\%) de um sol o franco-siltoso consolidado.

A concentração de sedimento (C) (Figura 1), similarmente à descarga sólida e taxa de desagregação, para todas as descargas líquidas (Q), foi sempre maior no $C R$ em relação à dos demais tratamentos. No tratamento CC, a C teve uma relação parabólica com o tempo de chuva, atingindo um valor máximo na segunda $Q$ aplicada $\mathrm{e}$ diminuindo posteriormente. Esse comportamento deveu-se, provavel mente, ao fato deter o escoamento atingido uma camada de sol o com maior resistência ao cisalhamento e, portanto, ser menos erodível, produzindo menor perda de solo e menor concentração de sedimento para determinada taxa de escoamento. Para os tratamentos PDS e PDC, praticamente não houve incremento na concentração de sedimento com o aumento na descarga líquida, em virtude da el evada consolidação e estruturação nos dois solos e da presença de cobertura no segundo solo.

Em síntese, a descarga sólida, a desagregação ea concentração de sedimento foram maiores no CR, intermediárias no CC e PDS, e menores no PDC,

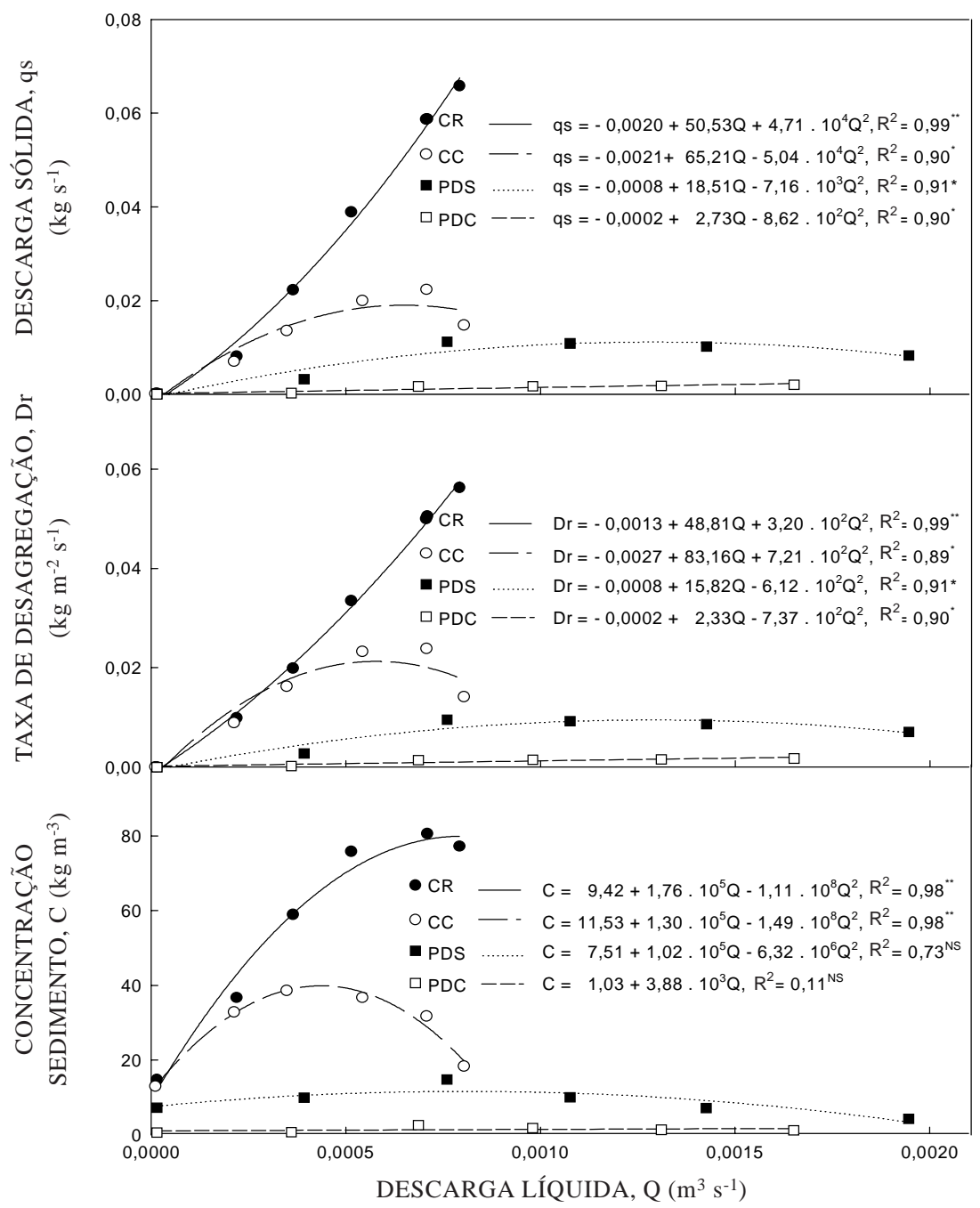

Figura 1. Descarga sólida (qs), taxa de desagregação em sulcos (Dr), concentração de sedimento (C) e descarga líquida $(Q)$, considerando diferentes preparos e consolidação do solo $(C R=$ convencional recente, $\mathrm{CC}=$ convencional consolidado, $\mathrm{PDS}=$ plantio direto sem palha, $\mathrm{PDC}=$ plantio direto com palha). $* *=$ significativo $(P<0,01)$; * = significativo $(P<0,05)$; NS = não-significativo. 
estando relacionadas com a consolidação, agr egação e cobertura do solo. Similarmente, King (1992) encontrou a seguinte seqüência decrescente na concentração de sedimentos em sulcos: preparo convencional recente, preparo convencional consolidado por pouco tempo, sistema plantio direto sem cobertura e plantio direto com cobertura.

O fator perda de carga (f) no PDC diminuiu exponencialmente com o aumento na descarga líquida (Q). Dos fatores de resistência no escoamento superficial, of pareceser o mel hor (Abrahams et al., 1986), o qual descreve perdas de carga resultantes de forças de cisal hamento do fluido com os resíduos vegetais e com a superfície do solo. Entre a segunda e a terceira Q (Quadro 3, Figura 2), o resíduo não foi mais responsável por essa perda (Quadro 3), pois foi suspenso e transportado para fora da parcela, semelhantemente ao observado por Foster (1982). Entretanto, o fato de a perda de carga no resíduo não chegar a zero significa que o resíduo poderia estar suspenso, mas não removido da parcela, atuando como um conjunto entrelaçado e preso ao solo (King, 1992). Nos demais tratamentos, não houve uma relação clara e significativa entref e Q.

O escoamento foi turbulento (Remaior que 2000), exceto nas descarga líquidas (Q) até $0,0004 \mathrm{~m}^{3} \mathrm{~s}^{-1}$, onde o escoamento foi transicional (Re entre 500 e 2000). A relação entre Re e Q foi linear (Figura 2). Graças à turbulência, o escoamento apresentou grande capacidade de provocar erosão.

O número de Froude (F) (Figura 2) indica que, apenas na primeira descarga no PDC com presença

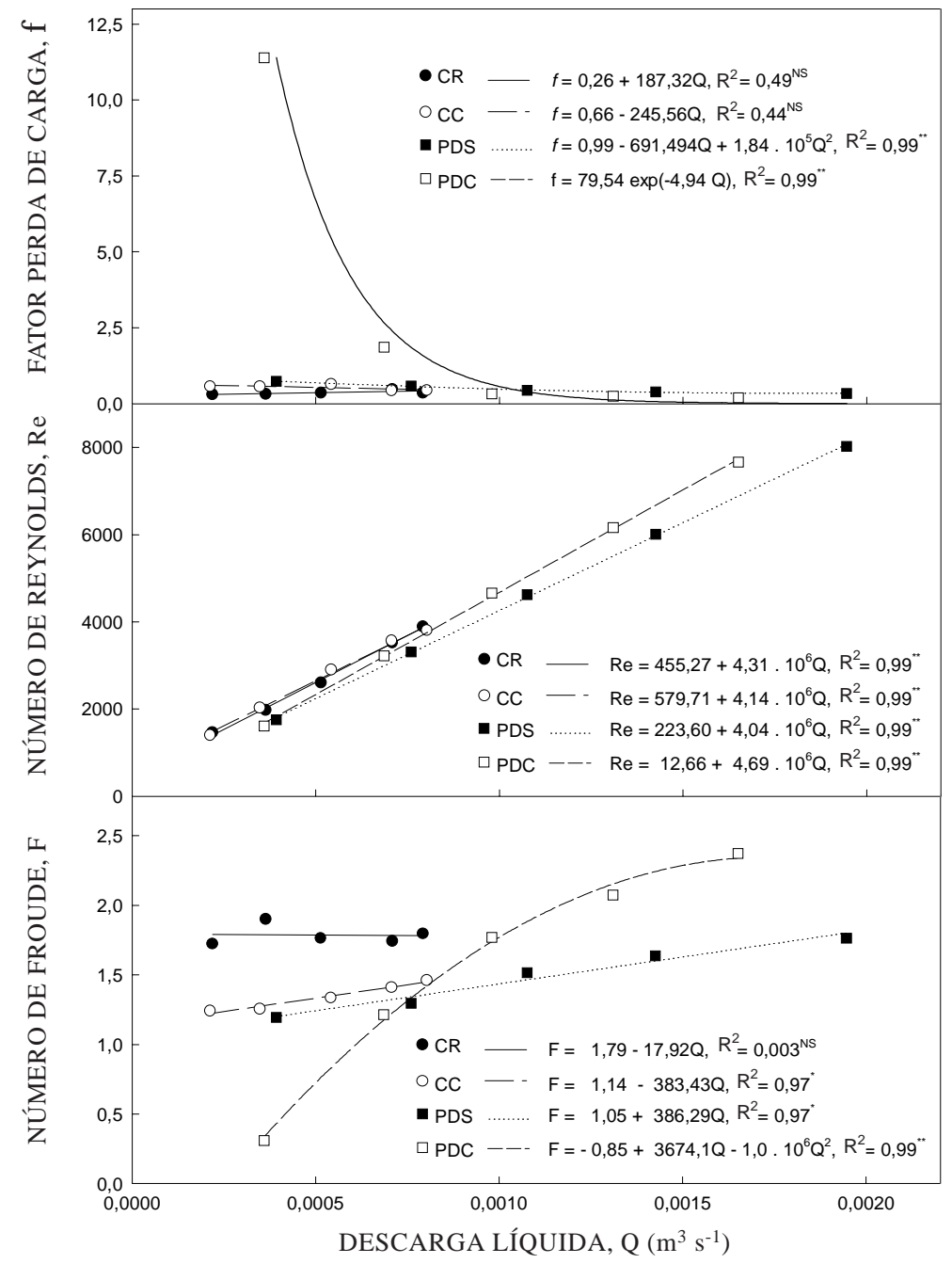

Figura 2. Fator perda de carga $(f)$, número de Reynolds $(R e)$ e de $F$ roude $(F)$ e descarga líquida $(Q)$, considerando diferentes preparos e consolidação do solo $(C R=$ convencional recente, $C C=$ convencional consolidado, PDS = plantio direto sem palha, PDC = plantio direto com palha). ${ }^{* *}=$ significativo ( $P<0,01)$; ${ }^{*}=$ significativo ( $\left.P<0,05\right)$; NS = não-significativo. 
Quadro 3. F ator perda de carga (f) e tensão cisal hante (t) do escoamento determinado para os tratamentos com sistema plantio direto

\begin{tabular}{cccccc}
\hline Descarga líquida & $\begin{array}{c}f_{\text {total }} \\
\text { PDS(1) }\end{array}$ & $\begin{array}{c}f_{\text {solo }} \\
\text { PDC }\end{array}$ & $\begin{array}{c}f_{\text {resíduo }} \\
\text { PDC }\end{array}$ & $\begin{array}{c}\tau_{\text {total }} \\
\text { PDC }\end{array}$ & $\begin{array}{c}\tau_{\text {solo }} \\
\text { PDC }\end{array}$ \\
\hline $\mathrm{m}^{3} \mathrm{~s}^{-1}$ & & & & $\mathrm{~N} \mathrm{~m}^{-2}-$ & 0,41 \\
0,0004 & 11,39 & 0,74 & 10,65 & 9,51 & 2,97 \\
0,0008 & 1,86 & 0,58 & 1,28 & 11,03 & $11,03(2)$ \\
0,0012 & 0,33 & 0,43 & $0(2)$ & 12,66 & 12,66 \\
0,0016 & 0,24 & 0,39 & 0 & 14,77 & 14,77 \\
0,0020 & 0,20 & 0,34 & 0 & & \\
\hline
\end{tabular}

(1) PDS $=$ plantio direto sem palha, PDC $=$ plantio direto com palha. ${ }^{(2)}$ Quando $f_{\text {total }}<f_{\text {solo, }}$, considerou-se $f_{\text {resíduo }}=0$ e $\tau_{\text {total }}=\tau_{\text {solo' }}$.

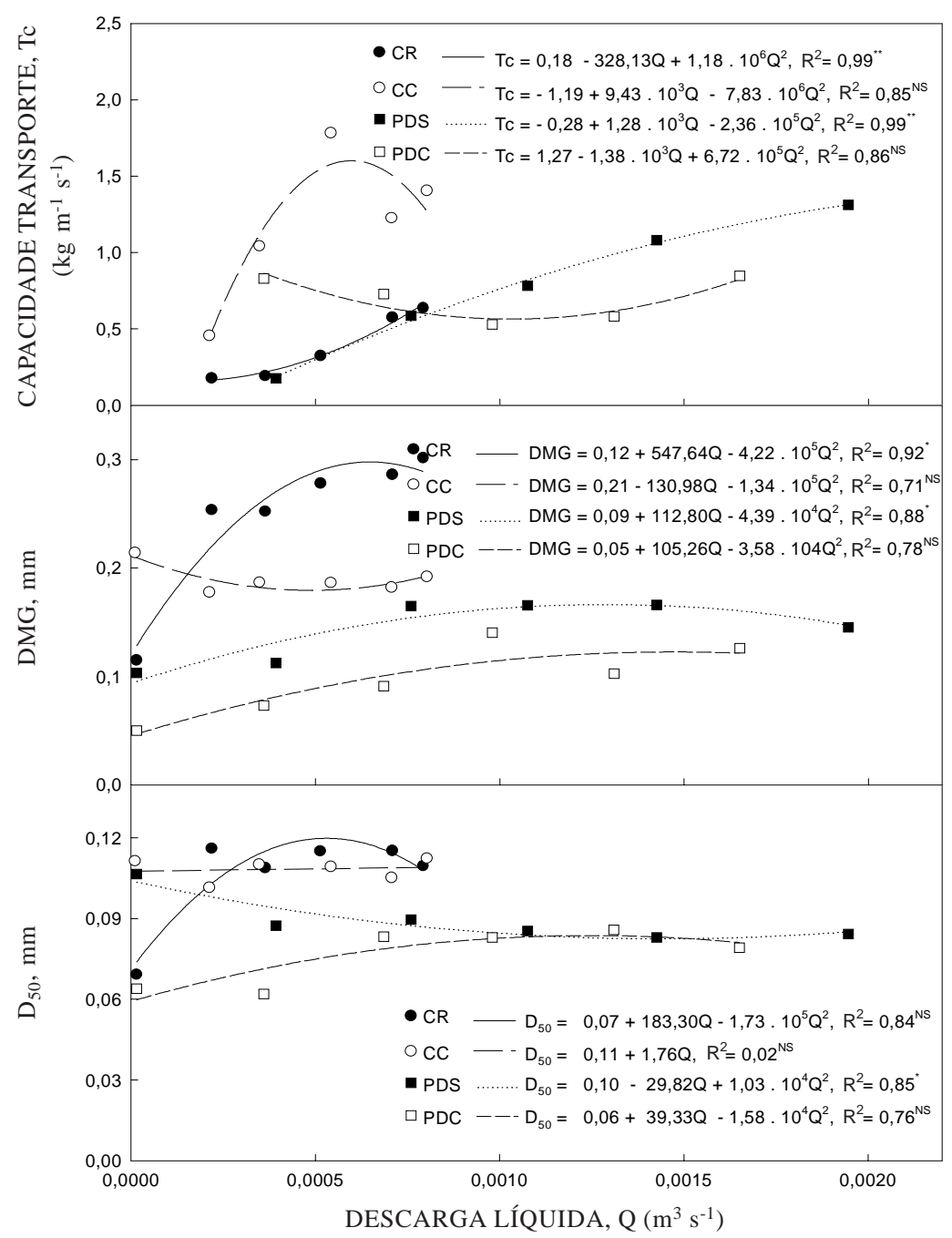

Figura 3. Capacidade de transporte de sedimento (Tc), diâmetro médio geométrico (DMG) e mediano $\left(D_{50}\right)$ de sedimentos erodidos e descarga líquida $(Q)$, consi derando di ferentes preparos e consolidação do solo (CR = convencional recente, $C C=$ convencional consolidado, $P D S=$ plantio direto sem palha, PDC = plantio direto com palha). ${ }^{* *}=$ significativo $(P<0,01) ; *$ significativo $(P<0,05)$; NS = nãosignificativo. 
de resíduos culturais, o escoamento se comportou como subcrítico, ou seja, tranqüil o sem formação de ondas (Morgan, 1995), enquanto, nas demais descargas noPDC, oregime passou a ser supercrítico em decorrência do aumento do escoamento e da remoção do resíduo. Nos demais tratamentos, o escoamento foi sempresupercrítico, indicando maior capacidade em provocar erosão.

A capacidade detransporte de sedimento (Tc) foi crescente com o incremento das descargas líquidas (Q) em todos os tratamentos (F igura 3), com exceção do PDC que apresentou um mínimo no momento da falha do resíduo, com um subseqüente incremento. A relação G/Tc (carga de sedimento/capacidade de transporte) foi pequena, sendo de 0,022 , para o CR; de 0,008, para oCC; de0,010, para oPDS, ede0,0005, para o PDC. Nesse caso, segundo (Elliot, 1988), o sedimento em transportenão reduziu a desagregação do solo.

A presença dos restos culturais sobrea superfície do solo no PDC proporcionou uma expressiva diminuição do DMG e $D_{50}$ dos sedimentos transportados pelo escoamento superficial nas diferentes descargas líquidas (Q) (Figura 3). Com exceção do CC, que teve um pequeno decréscimo inicial no tamanho do sedimento provavelmente devido ao arraste de partículas sol tas na superfície, o $D_{50}$ eo DMG aumentaram com o incremento da Q, atédeterminado valor, diminuindo posteriormente. Nos tratamentos PDC e PDS, houve pequeno aumento no DMG e $D_{50}$, tendendo a estabilizar-se nas maiores Q.

Com o incremento da $Q$, houve aumento na vel ocidade do escoamento e foram transportados sedimentos com maior tamanho, pois havia partículas separadas da massa do sol o edisponíveis ao transporte. Quando todos os sedimentos separados da massa já haviam si do transportados, o escoamento possivelmente dissipou mais energia para desagregar sedimentos e transportá-los, diminuindo o diâmetro dos sedimentos er osionados.

A tensão cisalhante $(\tau)$ e a potência $(\Omega)$ do escoamento apresentaram relação linear com a $Q$ em todos os tratamentos (Figura 4). As equações de regressão linear entre $\Omega$ e Q, para os diferentes tratamentos, foram praticamente paralelas e sobrepostas.

A tensão cisalhante representa uma média da tensão exercida na superfície do solo, pressupondo-

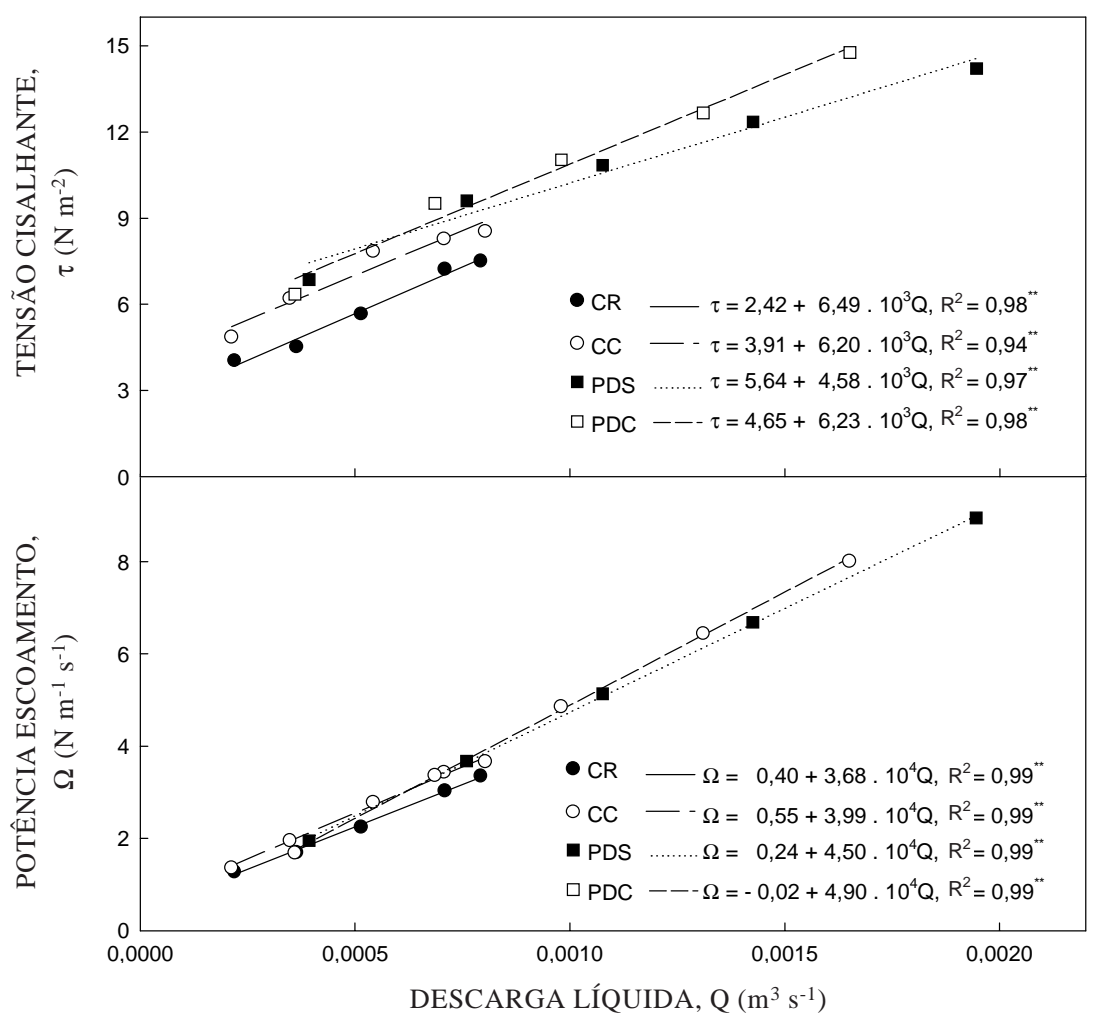

Figura 4. Tensão cisalhante $(\tau)$ e potência $(\Omega)$ do escoamento da descarga líquida $(Q)$, considerando diferentes preparos e consoli dação do solo ( $C R=$ convencional recente, $\mathbf{C C}=$ convencional consolidado, PDS = plantio direto sem palha, PDC = plantio direto com palha: $\tau$ e $\Omega$ atuantes no solo e no resíduo). $* *$ = significativo $(P<0,01)$. 
se o escoamento uniforme em toda a seção transversal do sulco; entretanto, isso não é correto dadas as variações no escoamento (Foster et al., 1984). Além disso, a quantificação da tensão cisalhante depende tanto da medição do raio hidráulico como da profundidade do sulco, que, no campo, são de difícil medição. Por essa razão, al guns autores (Bagnold, 1977; Nearing et al., 1991; Elliot \& Laflen, 1993) sugeriram usar a potência do escoamento em modelos de erosão, determinada com base na descarga sólida e declividade. Assim, neste trabalho, calcularam-se os dois parâmetros.

$\mathrm{O}$ valor de $\mathrm{Kr}$ determinado para este solo foi de $0,012 \mathrm{~kg} \mathrm{~N}^{-1} \mathrm{~s}^{-1}$ e o $\tau \mathrm{c}$ foi de $2,61 \mathrm{~N} \mathrm{~m}^{-2}$ (Figura 5). Esses val ores situam-se dentro dos limites de $\mathrm{Kr}$ de 0,0006 a $0,0453 \mathrm{~kg} \mathrm{~N}^{-1} \mathrm{~s}^{-1}$ e de $\tau \mathrm{c}$ de 0,4 a $6,6 \mathrm{~N} \mathrm{~m}^{-2}$ encontrados por Elliot et al. (1989) para solos dos Estados Unidos. No Brasil, Braida \& Cassol (1996) determinaram um $\mathrm{Kr}\left(0,0104 \mathrm{~kg} \mathrm{~N}^{-1} \mathrm{~s}^{-1}\right)$ semel hante e um $\tau c\left(4,81 \mathrm{~N} \mathrm{~m}^{-2}\right)$ maior para um Podzólico
Vermel ho-E scuro franco arenoso de textura similar ao solo deste estudo, enquanto Giasson \& Cassol (1996) quantificaram um Kr de 0,0077 $\mathrm{kg} \mathrm{N}^{-1} \mathrm{~s}^{-1} \mathrm{e}$ $\tau c$ de $1,13 \mathrm{~N} \mathrm{~m}^{-2}$ para um Plintossol o franco-argiloarenoso com teor de argila maior que o dos solos brasileiros supracitados. Esses resultados locais são ainda insuficientes para estabelecer uma relação entre os dois parâmetros de erosão em sulcos ( $\mathrm{Kr}$ e $\tau c)$ com propriedades do solo.

Considerando a potência do escoamento, o valor do parâmetro erodibilidade $\mathrm{Kr}_{\Omega}$ foi de $0,027 \mathrm{~kg} \mathrm{~N}^{-1} \mathrm{~m}^{-1}$ e a potência crítica do escoamento $\Omega$ c foi de $0,95 \mathrm{~N} \mathrm{~m}^{-1} \mathrm{~s}^{-1}$ (Figura 5). Em relação à tensão cisalhante, a potência do escoamento ajustou-se mel hor aos dados de desagregação.

O cál culo dos parâmetros erodibilidade e tensão cisalhante crítica para solos consolidados e com palha deve ser evitado. Vários estudos mostraram que desagregação não se correlacionou com tensão

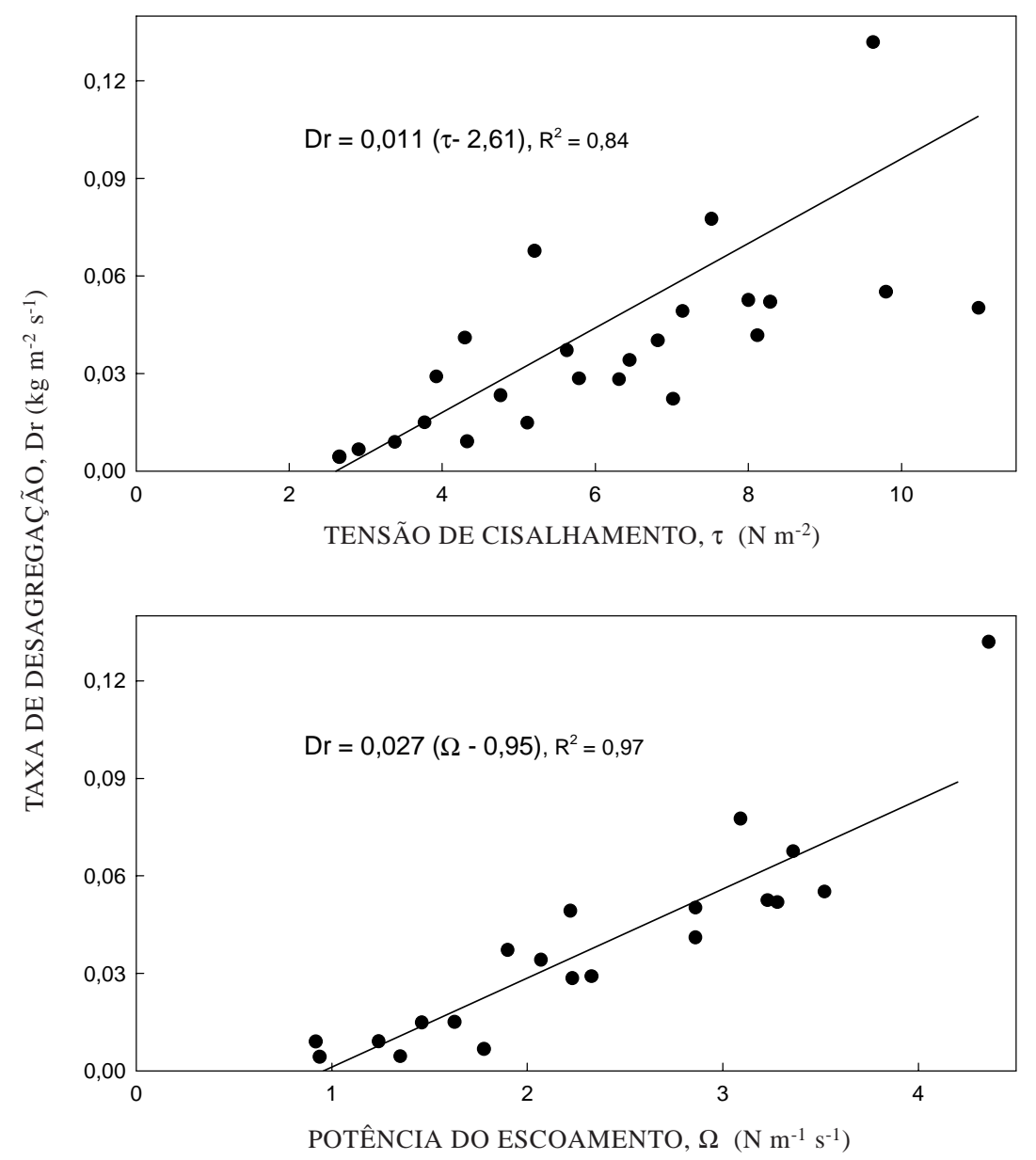

Figura 5. Relações entre taxa de desagregação (Dr) com tensão ci sal hante $(\tau)$ e potência $(\Omega)$ do escoamento para o tratamento convencional recente. Usaram-se somente as repetições cujo valores críticos de tensão cisalhante ou potência do escoamento foram positivos. 
cisalhante em solos consolidados ou com palha na superfície, obtendo valores inconsistentes, como $t_{c}$ negativo, ou grande variabilidadee baixo coeficiente de correlação (Brown et al., 1990; Norton \& Brown, 1992; Brown \& Norton, 1994), semel hantemente ao observado neste trabalho, embora possam haver exceções (Franti et al., 1985; King, 1992).

\section{CONCLUSÕES}

1. Para o ArgissoloVermelho-Amarelo distrófico arênico estudado, oval or básico da erodibilidade em sulcos $(\mathrm{Kr})$ foi de $0,012 \mathrm{~kg} \mathrm{~N}^{-1} \mathrm{~s}^{-1}$ e a tensão crítica decisal hamento $(\tau \mathrm{c})$ foi de2,61 $\mathrm{N} \mathrm{m}^{-2}$. Esses valores podem ser utilizados em model os físi cos queseparam a erosão em sulcos e em entressulcos.

2. A perda de solo, as taxas de desagregação em sulcos e o diâmetro mediano do sedimento erosionado em sulcos foram afetados pelo preparo e consolidação de solo, sendo mais intensos nos preparos convencionais (convencional recente e convencional com dois meses de consolidação) do que no plantio direto (tanto com palha quanto sem palha).

3. A consolidaçãoe o preparo do solo nãoinfluíram no regime do escoamento nos sulcos, o qual foi turbulento supercrítico, com exceção da menor descarga líquida aplicada, onde o regime de escoamento foi laminar subcrítico, para o plantio direto com palha, e laminar supercrítico, para os demais tratamentos.

\section{AGRADECIMENTOS}

Aos bolsistas de iniciação científica Márcio osé Silveira, Celso Gonçalves, Emerson Varlei Wohlemberg e Márcio Conceição, pelo auxílio nos trabal hos de campo e de laboratório.

\section{LITERATURA CITADA}

ABRAHAMS, A.D.; PARSON, A.J . \& LUK, S.H. Resistance to overland flow on desert hillslopes. J. Hydrol., 88:343-363, 1986.

ALVES, A.G.C.; COGO, N.P. \& LEVIEN, R. Relações da erosão do solo com a persistência da cobertura vegetal morta. R. Bras. Ci. Solo, 19:127-132, 1995.

ALVES, M.C. \& CABEDA, M.S.V. Infiltração de água em um Podzólico Vermelho-Escuro sob dois métodos de preparo, usando chuva simulada com duas intensidades. R. Bras. Ci. Solo, 23:753-761, 1999.
BAGNOLD, R.A. Bed load transport by natural rivers. Water Res. Res., 13:303-312, 1977.

BARCELOS, A.A.; CASSOL, E.A. \& DENARDIN , J .E. I nfiltração de água em um Latossolo Vermelho-Escuro sob condições dechuva intensa em diferentes sistemas demanejo. R. Bras. Ci. Solo, 23:35-43, 1999.

BERTOL, I.; COGO, N.P. \& LEVIEN, R. Comprimento crítico de declive em sistemas de preparos conservacionistas de solo. R. Bras. Ci. Solo, 21:139-148, 1997.

BRAIDA, J.A. \& CASSOL, E.A. Erodibilidade em sulcos e em entressulcos em um Podzólico Vermel ho-Escuro francoarenoso. R. Bras. Ci. Solo, 20:127-134, 1996.

BROWN, L.C. \& NORTON, L.D. Soil erosion from ridge-tillage systems. St. J oseph, American Society of Agricultural Engineering, 1990. (ASAE Paper 90-2563)

BROWN, L.C. \& NORTON, L.D. Surface residue effects on soil erosion from ridges of different soils and formation. Trans. Am. Soc. Agric. Eng., 37:1515-1524, 1994.

BROWN, L.C.; WEST, L.T.; BEASLEY, D.B. \& FOSTER, G.R. Rill erosion one year after incorporation of crop residue. Trans. Am. Soc. Agric. Eng., 33:1531-1540, 1990.

CASSOL, E.A. \& LIMA, V.S. Erosão em entressulcos em um Podzólico Vermelho-Amarelo do Rio Grande do Sul sob diferentes preparos do solo e manejo de resíduos culturais. In: REUNIÃO BRASILEIRA DE MANEJO E CONSERVAÇÃO DO SOLO E DA ÁGUA, 12., Fortaleza, 1998. Fortaleza, Sociedade Brasileira de Ciência do Solo, 1998. p.354-355.

CHOW, V.T. Open-channel hydraulics. New York, McGraw-Hill, 1959. 680p.

COGO, N.P.; MOLDENHAUER, W.C. \& FOSTER, G.R. Soil loss reductions from conservation tillage practices. Soil Sci. Soc. Am. J ., 84:368-373, 1984.

ELLIOT, W.J . \& LAFLEN, J .M. A process-based rill erosion model. Trans. Am. Soc. Agric. Eng., 36:65-72, 1993.

ELLIOT, W.J .; LIEBENOW, A.M.; LAFLEN, J .M. \& KOHL, K.D. A compendium of soil erodibility data from WEPP cropland soil field erodibility experiments 1987;88. West Lafayette, The Ohio State University/USDA-Agricultural Research Servive, 1989. p.A2-A25. (NSERL Report 3)

EMPRESA BRASILEIRA DE PESQUISA AGROPECUÁRIA EMBRAPA. Centro Nacional de Pesquisa de Solos. Sistema brasileiro de classificação de solos. Brasília, 1999. 412p.

FOSTER, G.R. Modeling the erosion process. In: HANN, C.T.; J OHNSON, H.P. \& BRAKENSIEK, D.L., eds. Hydrologic modeling of small watersheds. St. J oseph, American Society of Agricultural Engineering, 1982. p.297-380.

FOSTER, G.R.; HUGGINS, L.F. \& MEYER, L.D. A laboratory study of rill hydraulics: I. Velocity relationships. Trans. Am. Soc. Agric. Eng., 27:790-796, 1984.

FRANTI, T.G.; LAFLEN, J .M. \& WATSON, D.A. Soil erodibility and critical shear under concentrated flow. Am. Soc. Agric. Eng. Paper, 85-2033, 1985. 
GIASSON, E. \& CASSOL, E.A. Relações da erosão em sulcos com vazões aplicadas e doses de resíduos de trigo incorporados a um plintossol o franco-argil loarenoso. R. Bras. Ci. Solo, 20:117-125, 1996.

KING, K.W. Comparison of rill erodibility parameters as influenced by no-till farming. West Lafayette, Purdue University, 1992. 98p. (Tese de Mestrado)

MEYER, L.D.; FOSTER, G.R. \& RÖMKENS, M.J .M. Source of soil eroded by water from upland slopes. In: PRESE NT and prospective technology for predicting sediment yields and sources. Washington, USDA-Agricultural Research, 1975. p.177-189.

MORGAN, R.P.C. Soil erosion \& conservation. 2.ed. New York, Longman, 1995. 198p.

NEARING, M.A.; BRADFORD, J .M. \& PARKER, S.C. Soil detachment by shallow flow at low slopes. Soil Sci. Soc. Am. J ., 55:339-344, 1991.
NEARING, M.A.; WEST, L.T. \& BRADFORD, J .M. Consolidation of an unsaturated illitic clay soil. Soil Sci. Soc. Am. J ., 52:929934, 1988a.

NEARING, M.A.; WEST, L.T. \& BROWN, L.C. A consolidation model for estimating changes in rill erodibility. Trans. Am. Soc. Agric. Eng., 31:696-700, 1988b.

NORTON, L.D. \& BROWN, L.C. Time-effect on water erosion for ridge tillage. Trans. Am. Soc. Agric. Eng., 35:473-478, 1992.

SAS INSTITUTE. SAS/STAT users guide. Version 6.03. Cary, SAS Institute, 1988.

SCHÄFER, M.J .; REICHERT, J .M.; REINERT, D.J . \& CASSOL, E.A. Erosãoem entressul cos em diferentes preparos eestados de consolidação do solo. R. Bras. Ci. Solo, 25:431-441, 2001.

WEST, L.T.; MILLER, W.P.; LANGDALE, R.R.; BRUCE, R.R.; LAFLEN, J .M. \& THOMAS, A.W. Cropping system effects on interrill soil loss in the Georgia Piedmont. Soil Sci. Soc. Am. J ., 55:460-466, 1991. 